\section{Biological Defence}

\section{Introduction}

The protection of operational personnel under BW threat is a multifactorial discipline. In striking contrast to protection against nuclear and chemical weapons there exists the potential to minimise the effects of BW through employment of medical countermeasures. The combined use of medical countermeasures, physical protection, warning, detection and hazard management provides the basis of defence.

\section{MEDICAL COUNTERMEASURES}

\section{Immunoprophylaxis}

Active Immunoprophylaxis

Vaccination is an important practical means of providing continuous protection against BW threats prior to, as well as during,

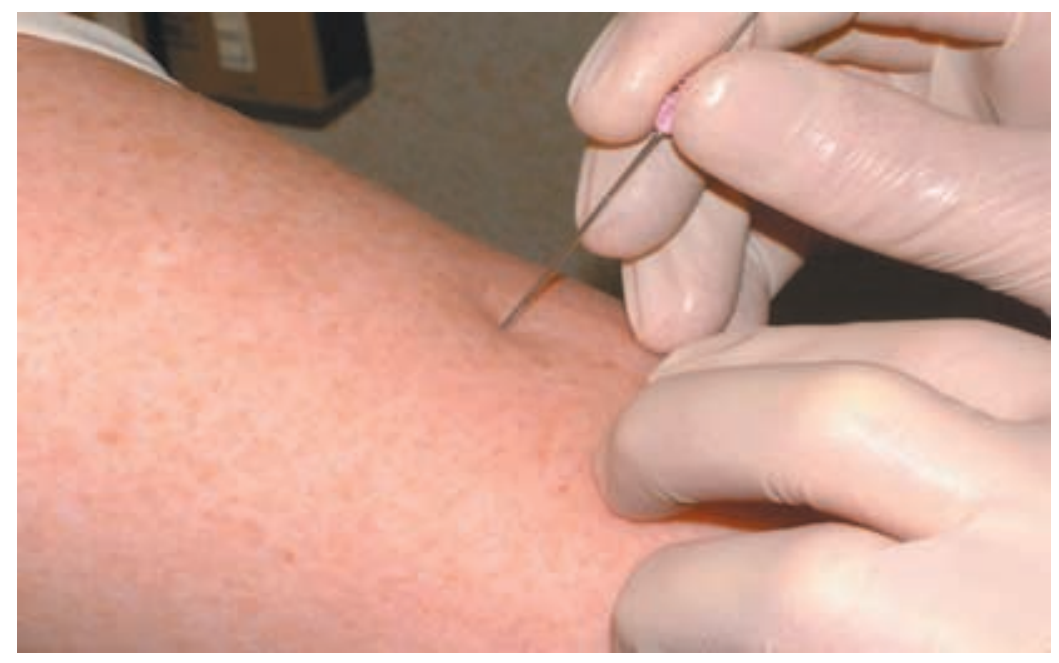

Fig 1. Smallpox vaccination.

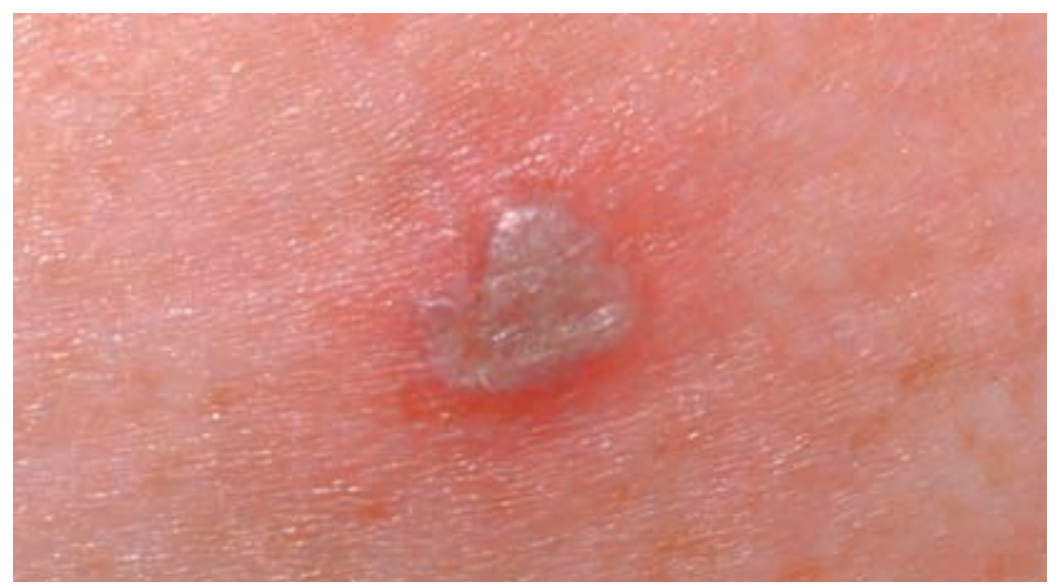

Fig 2. Smallpox vaccination site at day 7.

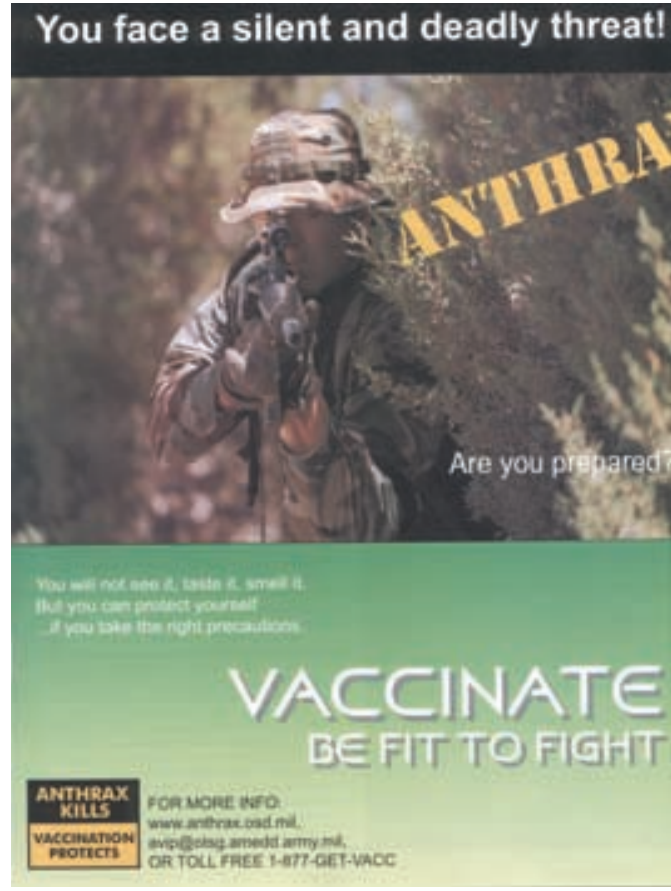

Fig 3. US anthrax vaccination poster.

hostile actions. Vaccines against a number of potential BW agents are available. Many of these vaccines were developed for the protection of laboratory workers or individuals working where the target diseases are endemic.

In a BA attack the number of infectious or toxin units to which an individual is exposed may be greater than in the case of natural exposure. Exposure by inhalation may represent an unnatural route of infection with many BA. The efficiency of protection afforded by most vaccines is based on natural inoculum size and exposure. Vaccines, which are generally considered to be effective under natural circumstances, may not provide a similar degree of protection to individuals exposed to BA attack.

An appropriate immunisation policy is essential. Vaccines are BA specific and do not provide immediate protection. Not all vaccines can be administered simultaneously; therefore to prevent the logistic problems caused by in-theatre vaccination prior immunisation is essential.

If a vaccination programme is required in theatre the possibility of adverse reactions from vaccination and the concomitant degradation of operational efficiency must be taken into account. 
Passive Immunoprophylaxis

For some BA, the only available medical countermeasure might be specific antiserum. Under certain conditions, passive immunoprophylaxis with immunoglobulin products might be considered. Use may be limited by lack of adequate sources and quantities of material, limited duration of protection, and the risk of serum sickness associated with antisera of animal origin. However, recent scientific advances in products for immunoprophylaxis (for example, human monoclonal antibodies, "despeciated" equine or ovine antisera) are making this option technically more attractive.

\section{Chemoprophylaxis}

Chemoprophylaxis using appropriate drugs (antibacterial, antiviral etc) offers additional protection in the setting of a BW threat. If an attack is felt to be imminent, or is known to have occurred, Command directed chemoprophylaxis would be appropriate for all personnel in the area. However, it is impractical and wasteful to place everyone located in a potential target area on prolonged, routine prophylactic antimicrobial therapy in the absence of such a threat condition.

For bacterial agents antibiotics should be administered as soon as possible following exposure. Initiation of chemoprophylaxis during the incubation period is always worthwhile, however the earlier the antibiotic is given the greater is the chance of preventing disease. In some cases (for example, inhalation anthrax), post exposure vaccination must be given in addition to antibiotics to personnel previously unvaccinated in order to prevent late onset of disease when antibiotics are withdrawn.

Consideration should be given to the possibility of the interaction between drugs in multi-drug regimens that address the multiple elements of force protection.

\section{PHYSICAL PROTECTION}

Individual Protection Equipment (IPE)

Respiratory Protection. In the presence of any BW inhalation hazard respiratory protection is essential. Currently fielded respirators (NBC protective mask) equipped with standard NBC filter canisters will provide a high degree of protection against particles greater than 0.3 micrometers in size. Other forms of protection, for example, self-contained breathing apparatus are available and may be fielded to meet particular conditions.

Dermal Protection. Intact skin provides an excellent barrier against BA, however any skin abrasions or exudative inflammation must be covered. In some instances it may be necessary to protect the mucous membranes of the eye. IPE clothing employed against NBC agents will protect against skin contamination with BA, although standard uniform clothing affords a certain degree of protection against dermal exposure to the surfaces covered.

Casualties. Casualties unable to continue wearing IPE in a BA contaminated area, should be held and/or transported using containment measures to protect the patient against BA exposure. Contagious patients should be held in an uncontaminated area by implementation of barrier measures able to prevent environmental spread of the BA.

\section{Collective Protection}

A dedicated hardened or unhardened shelter equipped with an air filtration unit (AFU) providing overpressure gives only limited collective protection (COLPRO) for personnel in the biologically contaminated environment. Appropriately decontaminated casualties will enter COLPRO for further treatment.

COLPRO is the most effective method for protecting patients and the medical facility during the primary BW attack. Patients whose illness is thought to be the result of a BW attack, or those who are thought to have a transmissible disease, will necessarily be cared for using barrier nursing techniques. The environment within COLPRO encourages cross-infection between casualties and staff and it may be appropriate to care for these patients outside COLPRO.

\section{HAZARD MANAGEMENT}

\section{Environmental Health Measures}

NATO Standardisation Agreement (STANAG 2048) 'Chemical Methods of Insect and Rodent Control' and STANAG 2136 'Minimum Standards of Water Potability in Emergency Situations' are of use in provision of environmental health measures.

Hygiene. The importance of effective hygiene and sanitation in a biological operations environment cannot be overemphasised. One of the primary responsibilities of all personnel is to ensure that standards of hygiene are maintained even in the most difficult circumstances. Personal hygienic measures such as frequent and adequate washing with soap and water, regular changes with laundered clothing, use of clean toilets and field latrines and post-defaecation hand-washing should be emphasised.

Food and Water Sanitation. High standards of food preparation and water purification are essential, as is the protection of food and water supplies from incidental airborne contamination or sabotage.

Waste Disposal. Standard methods of disinfection and waste disposal are effective in the context of BW. Particular care and 
attention should be paid to handling and disposal of clinical waste.

Vector Control. Since BA may be spread by mechanical means or natural vectors, rodents and arthropods must be effectively controlled.

\section{Contamination Control}

Casualty decontamination for BA can only occur immediately after a known BW attack. Often, this will not be the case and will therefore not be an issue. It is recommended that outer garments are removed and all areas of the casualty to be washed down with a $0.5 \%$ sodium hypochlorite solution. BA decontamination should follow CW decontamination and immediate life-saving resuscitation. 